\title{
PROYECCIÓN INVESTIGATIVA. HACIA UN NUEVO ENFOQUE DE LA CARRERA DE EDUCACIÓN BÁSICA DE LA UNIVERSIDAD NACIONAL DE EDUCACIÓN.
}

\author{
INVESTIGATIVE PROJECTION. A NEW APPROACH TO RACE \\ BASIC EDUCATION NATIONAL UNIVERSITY OF EDUCATION.
}

\author{
Dr. Rolando Juan Portela Falgueras (PhD) \\ Profesor-investigador Titular Principal 1. Director de la Carrera de Educación Básica \\ Universidad Nacional de Educación (UNAE) \\ e-mail: rolando.portela@unae.edu.ec
}

\author{
Dr. Luis Enrique Hernández Amaro (PhD) \\ Profesor-investigador Principal 1 \\ Universidad Nacional de Educación (UNAE) \\ e-mail: luis.hernandez@unae.edu.ec
}

\author{
Dra. Ana Delia Barrera Jiménez (PhD) \\ Profesora-investigadora Titular Principal 1. Directora de Postgrado \\ Universidad Nacional de Educación (UNAE) \\ e-mail: ana.barrera@unae.edu.ec \\ Recibido:
}

Aprobado: 20 de octubre de 2016

\section{RESUMEN}

En correspondencia con los nuevos objetivos de desarrollo de los profesionales ecuatorianos, resulta necesario el establecimiento de modificaciones en las estructuras organizativas y el sistema de organización del proceso educativo de la Carrera de Educación Básica (EB) de la Universidad Nacional de Educación (UNAE), en tanto que en ella se aprecian incoherencias y dificultades. Estas modificaciones se proyectan desde un nuevo enfoque que, como deber ser de la carrera, orientan y regulan su desarrollo en las condiciones actuales de la Universidad, a la vez que constituyen el punto de partida para proyectar y ejecutar una investigación orientada a lograr ese nuevo enfoque en el proceso de formación profesional de los docentes de Educación General Básica. Desde un proyecto investigativo en marcha se debe contribuir a la solución del problema de investigación, en tanto posibilitará poder disponer de una sistematización teórica de la formación del profesional de la carrera y de su mejoramiento en las condiciones del contexto ecuatoriano. A partir de la operacionalización de la variable "formación del profesional de la EGB en las condiciones de la UNAE", se elaborarán los instrumentos para el diagnóstico del estado actual de este proceso y, a partir de sus resultados, se propondrá una nueva concepción para el mejoramiento de la formación del profesional en la carrera. EL proyecto contribuirá a reforzar teóricamente criterios pedagógicos relevantes presentes en el 
Modelo Pedagógico de la UNAE y, desde el punto de vista práctico, aportará una estrategia para la implementación de la concepción resultante.

Palabras Claves: Caracterización del deber ser de la Carrera de Educación Básica, procesos sustantivos universitarios, formación profesional, proyecto de investigación.

\section{SUMMARY}

In line with the new objectives of development of Ecuadorian professionals, establishing changes in organizational structures and the system of organization of educational process Career Basic Education (EB) of the National University of Education (UNAE) it is necessary, while it appreciated inconsistencies and difficulties. These changes are projected from a new approach, as must be of the race, guide and regulate its development in the current conditions of the university, while constitute the starting point for designing and implementing a strategy to achieve research that new focus on the process of training of teachers of basic general education. From a research project underway should contribute to the solution of the research problem, as it will allow to have a theoretical systematization of training professional career and his improvement in the conditions of Ecuadorian context. From the operationalization of the variable "professional training of EGB in the conditions of the UNAE" instruments for the diagnosis of the current state of this process will be developed and, based on their results, a new approach is proposed for improving professional training in the race. The project will help strengthen theoretically relevant educational criteria present in the pedagogical model of UNAE and from the practical point of view, provide a strategy for the implementation of the resulting conception.

Keywords: Characterization of what should be Race Basic Education, substantive processes university, vocational training, research project.

\section{Introducción}

Dado el alto valor que le es inherente a la educación, la Universidad Nacional de Educación (UNAE) constituye un componente estratégico para el logro de la transformación social a que se aspira en el Ecuador.

La educación es considerada como “... el instrumento para la construcción de un conocimiento emancipador, a través de la formación académica y profesional integral, desde una visión humanista y científica que incorpore los saberes y las culturas de nuestro pueblo". (1)

(...) la UNAE es un eje central para la transformación de la calidad del sistema educativo, procurando la formación de docentes y profesionales de la educación de la máxima excelencia en todas sus dimensiones: cognitivas, afectivas, personales, sociales y profesionales. (1)

Consecuente con ese criterio orientador, la carrera de Educación Básica (EB) de la UNAE pone en centro de su atención al estudiante y futuro docente,

(...) como un sujeto ético, innovador y transformador de la realidad educativa. La finalidad de esta carrera es formar profesionales con compromiso ético y docentes de excelencia para que posean la capacidad de investigar, analizar, planificar, evaluar y retroalimentar planes, programas, proyectos y estrategias educativas y curriculares. (2)

Esta carrera se propone la formación de profesionales de la educación altamente calificados para desempeñarse como docentes de la Educación General Básica (EGB), en cualquier sector y ámbito del Ecuador. Esa alta calidad en el desempeño profesional de sus egresados ha de expresarse en sus posibilidades para la resolución de problemas, la investigación sobre la práctica, la permanente reflexión individual y con otros, la sólida formación interdisciplinar y un alto compromiso ético con la educación como derecho.

En la carrera los procesos formativos se orientan al logro de objetivos vinculados al conocimiento y los saberes; vinculados a la pertinencia; vinculados al aprendizaje; y, los vinculados a la ciudadanía integral. En estos últimos la carrera se propone contribuir al fortalecimiento del sistema educativo ecuatoriano, con el aporte de profesionales docentes altamente calificados. 
Desde estos objetivos, la carrera se orienta al desarrollo de competencias profesionales: investigativas, docentes y de gestión pedagógica, que aseguren el desempeño de la profesión; ello determina el requerimiento de otorgar un nuevo significado a la formación del profesor de EGB en la UNAE, a partir del establecimiento de estructuras curriculares, procesos y estilos más dinámicos, democráticos, integrados y creativos, que, en su integralidad, constituyan un nuevo enfoque de la formación del profesional para este sistema educativo en las condiciones de la UNAE. Para ello se ha concebido un proyecto de investigación que aporte una concepción pedagógica que contribuya al perfeccionamiento de dicho proceso formativo a partir del logro de ese nuevo enfoque.

\section{Desarrollo}

A la Universidad Nacional de Educación (UNAE), cuya misión es: "Contribuir a la formación de talento humano del sistema educativo para la construcción de una sociedad justa, equitativa, libre y democrática, generando modelos educativos de excelencia caracterizados por su rigor científico, enfoque de derechos y de interculturalidad" (3), le corresponde prestar especial atención a la marcha de la formación de los profesionales de la educación y encausar dicho perfeccionamiento en la medida que se precisan e incrementan las regulaciones y criterios de calidad establecidos en el Sistema de Educación Superior del país.

Por su importancia para la UNAE y la nación, le corresponde a los directivos, profesores y estudiantes hacer realidad ese proceso en la carrera de EB y concretarlo en el accionar consciente de cada uno de sus profesores, en correspondencia con la guía orientadora del objetivo general de esa carrera:

Formar profesionales docentes de excelencia para la enseñanza-aprendizaje de los niños/as y jóvenes del sistema de Educación General Básica, capaces de investigar, analizar, fundamentar, planificar, gestionar, evaluar y retroalimentar planes, programas, proyectos y estrategias educativas y curriculares interculturales, orientadas a la prevención y resolución de los problemas en el ámbito educativo, en el marco de la equidad, la inclusión y la innovación pedagógica y social. (3).

Dado el alto valor que le es inherente a la educación, la UNAE y, en particular su Carrera de EB, constituyen un componente estratégico para el logro de la transformación social a que se aspira en el Ecuador. Ello demanda prestar especial atención al proceso de formación profesional pedagógica y al logro de un alto desarrollo de las competencias profesionales en sus egresados.

Al referir el desempeño profesional como parámetro a lograr con el proceso formativo de la carrera, resulta oportuno una aproximación conceptual de este término en su relación con el de profesionalización.

Un informe referido a la formación del profesor del Ministerio de Educación y Ciencia de España define el desarrollo profesional del docente como:

(...) cualquier intento sistemático de cambiar la práctica, creencias y conocimientos profesionales del docente, hacia un propósito de mejora de la calidad docente, investigativa y de gestión. Este concepto incluye el diagnóstico de las necesidades actuales y futuras de una organización y sus miembros, y el desarrollo de programas y actividades para la satisfacción de estas necesidades". (4)

Esta definición resalta algunos aspectos importantes. Desde ella se aprecia que el desarrollo profesional

(...) debe entenderse como un proceso planificado, de crecimiento y mejora, en relación con el propio conocimiento, con las actitudes hacia el trabajo, con la institución, y buscando la interrelación entre las necesidades de desarrollo personal y las de desarrollo institucional y social, (...) es pues, la construcción de la identidad profesional que procura incrementar la satisfacción en el ejercicio de la profesión a través de una mayor comprensión y mejora del desempeño profesional". (5)

Estos criterios en alguna medida coinciden con la definición de profesionalización pedagógica que realizan Añorga J y Valcárcel N. Estos autores definen la profesionalización como 
(...) el proceso pedagógico profesional permanente que tiene su génesis en la formación inicial del individuo en una profesión, que lleva implícito un cambio continuo obligatorio a todos los niveles, con un patrón esencialmente determinado por el dominio de la base de conocimientos, propios de la disciplina específica de la profesión que ejerce, que tiene un factor humano que debe reaccionar de forma correcta en su enfrentamiento con la comunidad y avanzar para ser capaz de hacer un ajuste conveniente con las innovaciones de variables intercambiables que infieren en un entorno social". (6)

En este sentido, y particularizando en el proceso de profesionalización del docente, Pérez A M plantea que es:

(...) el proceso pedagógico de formación y autoformación del profesional, que tiene su génesis en la formación inicial vocacional del individuo y llega hasta la formación permanente y continua del personal en ejercicio. Lleva implícito un cambio continuo y ascendente determinado por el dominio de los contenidos propios de las disciplinas de la profesión que ejerce con un componente esencialmente humano (...) en él se dan tres elementos constitutivos importantes: el conocimiento teórico, la profesión y la ciencia en particular". (7)

Los investigadores referidos coinciden en que la profesionalización pedagógica de los profesores es un proceso permanente que tiene su génesis en la formación inicial del individuo en su profesión como docentes, que implica un cambio continuo, ascendente y obligatorio, con un patrón determinado por el dominio de los conocimientos propios de la disciplina específica de la profesión que ejerce, y que tiene un factor humano en estrecho vínculo con la comunidad en un entorno social determinado. De igual forma, la profesionalización lleva asociada la categoría desempeño y en él resulta importante considerar además de los conocimientos, las habilidades, cualidades, motivaciones, actitudes y los valores que son indispensables para que su desempeño profesional se manifieste satisfactoriamente, en correspondencia con las exigencias de la sociedad.

Para la investigadora Añorga J, el desempeño profesional es:

(...) la capacidad de un individuo para efectuar acciones, deberes y obligaciones propias de su cargo o funciones profesionales que exige un puesto de trabajo. Esta se expresa en el comportamiento o la conducta real del trabajador en relación con las otras tareas a cumplir durante el ejercicio de su profesión". (8)

Esta autora personaliza el desempeño profesional a partir de un conjunto de cualidades propias de un individuo, capaz de reconocer que solo a través del desarrollo de acciones claras que van más allá de lo que él conoce y de lo que el puesto de trabajo exige hacer.

Otros autores refieren un concepto más elaborado donde se evidencia la necesidad del dominio de elementos cognitivos para el desarrollo del desempeño profesional pedagógico y la vinculación de este con el contexto.

Moreno V define el desempeño profesional pedagógico como:

(...) la actuación real de los profesores en la práctica docente, en donde se manifiesta en forma actualizada el dominio de un determinado campo del saber y de estrategias pedagógicas, conocimiento de los sujetos y sus interacciones personales, y conocimiento de los contextos en que tiene relación, en los marcos de responsabilidades docentes preestablecidas y asumiendo una actitud ética, de ayuda y colaboración a la institución escolar, a la comunidad educativa durante el proceso educativo en las condiciones sociales, ambientales, materiales y políticas existentes. (9)

Por su parte, (10) lo define como:

(...) el proceso que se desarrolla a través de las relaciones de carácter social que se establecen en la aplicación de métodos para el cumplimiento de su contenido de trabajo; en correspondencia con los objetivos de la actividad en que participa y el logro de un resultado que evidencia el mejoramiento profesional, institucional y social alcanzado, la atención a la educación de su competencia laboral y la plena comunicación y satisfacción individual al ejecutar sus tareas con cuidado, precisión, exactitud, profundidad, organización y rapidez. (10) 
En las definiciones aportadas se le confiere un significativo valor al carácter social del desempeño profesional, lo que se asume en el proyecto de investigación orientado al perfeccionamiento de la carrera de EB de la UNAE, al considerar que el profesional se desempeña en dependencia de las características y exigencias sociales en que se desarrolla. De forma explícita o implícita estas definiciones refieren que en el desempeño profesional de los docentes se revelan sus conocimientos sobre un área determinada, así como de los fundamentos teóricos y prácticos fundamentales de las ciencias pedagógicas; el dominio de habilidades vinculadas con la profesión docente; así como sus actitudes y valores profesionales.

Desde las definiciones de desempeño profesional pedagógico analizadas, se pudo determinar los rasgos comunes y se precisaron las principales regularidades de este proceso, las que sirvieron de base para la elaboración de una definición operativa de desempeño profesional pedagógico de los profesores en formación en la carrera de EGB de la UNAE.

- Es un sistema que revela la idoneidad del profesional de la educación para ejecutar las acciones propias de sus funciones.

- Es un proceso que comienza con la formación profesional y que implica el desarrollo gradual e integrado de sus conocimientos, habilidades, actitudes, valores y otros recursos propios de la profesión docente, que regulan su actuación y que se manifiesta en la práctica en sus modos de actuación.

- Se desarrolla en la práctica social, construido desde la superación sistemática del docente para lograr su mejoramiento humano y profesional pedagógico.

- Permiten valorar la práctica en sus múltiples expresiones y evaluar la eficiencia del trabajo del profesor en cualquier contexto social, así como promover su transformación en un entorno concreto.

Este proceder metodológico permitió precisar que el desempeño profesional pedagógico del profesor en formación en la carrera de EB de la UNAE, es el proceso en el que se expresa la integración dialéctica de los conocimientos, habilidades, actitudes y valores propios de la profesión docente, vinculados al desarrollo curricular, la gestión del aprendizaje que realiza en la dirección del proceso docente educativo del Sistema de EGB en todos sus niveles y contextos, su profesionalización pedagógica y su compromiso ético en la formación integral de los niños (as) y jóvenes que transitan por este nivel educativo, en correspondencia con las nuevas exigencias planteadas por la Ley Orgánica de Educación Inclusiva y el resto de las normativas del Ministerio de Educación del Ecuador.

Con esta definición se reconoce el carácter social con que se concibe el proceso formación profesional en la carrera, para el logro de egresados caracterizados por un adecuado desempeño profesional pedagógico en el ejercicio de sus funciones docentes.

En estrecha relación con el desarrollo de las competencias profesionales pedagógicas que se han de expresar en el desempeño profesional de los futuros maestros de EGB, el proyecto defiende la necesidad de integrar coherentemente los tres procesos sustantivos propios del proceso educativo de la educación superior: el académico, el investigativo y el de las prácticas pre-profesionales, de modo que en su organización y dinámicas internas, permitan la apropiación de los modos de actuación profesional.

Al profundizar en este criterio, se valora importante significar que "La educación superior debe tomar en cuenta que la formación del profesional implica la profundización, dignificación y producción permanente de las dinámicas y dimensiones de la condición humana." (11)

Las categorías analizadas hacen referencia a procesos universitarios que, en sus interrelaciones sistémicas, deben ser valorados y asegurados como directrices de transformación de la formación profesional en la carrera como parte de su perfeccionamiento. Así, el deber ser de la docencia, en integración con la investigación científica y la vinculación con la sociedad, en correspondencia con el contexto de la carrera, ha de tener en cuenta los cambios que actualmente operan en los horizontes epistemológicos del conocimiento, las nuevas tendencias de la educación superior a nivel internacional y latinoamericano, así como las principales reformas y requerimientos planteados a las universidades ecuatorianas. 
La concepción pedagógica que se prevé lograr con el proyecto de investigación se orienta hacia ese deber ser de estos procesos de acuerdo a dichos requerimientos; ello representará un nuevo enfoque u horizonte epistemológico a asumir como criterio básico para el perfeccionamiento de la carrera de EB en la UNAE y la formación profesional de sus egresados. El mejoramiento e integración de los procesos universitarios en la formación de los futuros profesionales de EGB constituye un importante cambio respecto a las formas con que tradicionalmente se ha concebido y concibe el proceso de formación de los profesionales de la educación en el país.

Enmarcados en estos procesos básicos, en estrecha relación entre sí y con vinculación con la colectividad, la concepción pedagógica que se derive del proyecto debe favorecer una mayor coherencia en el accionar pedagógico y didáctico de la carrera de EB de la UNAE, y así contribuir al logro real de sus objetivos específicos (3).

De este modo, el proyecto debe contribuir al desarrollo de competencias profesionales que se expresen en el desempeño profesional pedagógicos de sus egresados: investigativas, docentes y de gestión pedagógica, de acuerdo a los requerimientos del sistema de EGB. Para ello resulta necesario precisar el objeto de la profesión y las particularidades de estas competencias en cada uno de los ciclos.

En un intento de que el proyecto parta de un acercamiento inicial a ese nuevo enfoque o deber ser de la carrera, se correlacionaron las competencias básicas y profesionales establecidas en el Modelo Pedagógico de la UNAE con los núcleos problémicos previstos como elementos de integración para cada uno de sus ciclos. Estos núcleos son los que deben dar respuesta a los problemas profesionales que, como guía para la formación de los docentes de EGB, constituyen las situaciones inherentes al objeto de la profesión que deberán resolver durante el desempeño de su labor educativa profesional. Gradualmente los estudiantes de la carrera deberán enfrentarlos con la ayuda de sus tutores y profesores, en la medida que se incrementa el grado de independencia y creatividad según el núcleo problémico de cada ciclo y, en consecuencia, desarrollen las competencias básicas y profesionales que los debe caracterizar para ejercer de manera satisfactoria su función docente.

Existen diferentes posiciones acerca los de resultados de aprendizaje, los cuales se asumen como "descripciones de lo que los estudiantes son capaces de demostrar en términos de conocimientos, destrezas y actitudes, una vez completado un programa." (12). En relación con estos descriptores, las competencias se asumen como "la demostración efectiva y creativa y el despliegue del conocimiento y las destrezas en situaciones humanas. Dichas situaciones pueden comprender situaciones cívicas y sociales generales, como también profesionales de índole específico." (12)

(...) Al utilizar el término de cualidades, competencias o pensamiento práctico estamos aludiendo a sistemas de comprensión y actuación, y que, por tanto, incluyen saber pensar, saber decir, saber hacer y querer pensar, decir y hacer. El compromiso y la implicación activa del docente es clave en el desarrollo profesional e incluye evidentemente aspectos racionales y emotivos, conocimiento explícito y conocimiento tácito, técnicas y habilidades concretas y estrategias y modelos teóricos. (1)

Como se considera en el Modelo Pedagógico de la UNAE, el docente debe desarrollar las competencias que pretende desarrollar en sus estudiantes. Por ello, a lo largo de la carrera, todos los docentes deberán contribuir, según los núcleos básicos y desde las diferentes asignaturas de cada uno de los campos de formación, al desarrollo de las competencias básicas siguientes: (1)

- Capacidad de utilizar y comunicar de manera disciplinada, crítica y creativa el conocimiento. La mente científica y artística.

- Capacidad para vivir y convivir en grupos humanos cada vez más heterogéneos. La mente ética y solidaria.

- $\quad$ Capacidad para pensar, vivir y actuar con autonomía. La construcción del propio proyecto vital. La mente personal.

Estas competencias deben complementarse e integrarse con las competencias profesionales que se deben formar en los futuros profesionales de la EGB: (1)

- Capacidad para comprender y diagnosticar sujetos, situaciones, procesos y sistemas educativos. El docente se encuentra ante un grupo de estudiantes en una edad determinada, en 
una escuela, un contexto, un barrio y una comunidad y tiene que ser capaz de diagnosticar, en equipo con los demás compañeros de esa escuela o de esa comunidad, los sujetos, las situaciones, los procesos y los sistemas con los que se enfrenta, con sus fortalezas y debilidades, posibilidades, condiciones y dificultades singulares.

- Capacidad para diseñar, desarrollar y evaluar de manera personalizada el currículum. El docente de la era digital ha de diseñar, planificar, desarrollar y evaluar el curriculum de manera personalizada, para intentar ayudar a que cada aprendiz construya sus propias competencias y cualidades humanas de manera singular. La tarea del docente debe orientarse a la definición y planteamiento de situaciones en las cuales los alumnos puedan construir, modificar y reformular conocimientos, actitudes, habilidades, emociones y valores. Eso significa evidentemente la necesidad de plantearse la concreción de las líneas curriculares genéricas en problemas, situaciones, proyectos y casos en cuyo análisis y solución deben implicarse activamente los aprendices.

- Capacidad para diseñar y construir contextos y comunidades de aprendizaje. Lo que hoy día parece cada vez más claro desde el ámbito de la Psicología, de la Neurociencia, y de la Didáctica, es que los aprendizajes relevantes y permanentes son aquellos que se desarrollan como subproductos de vivir contextos educativos vinculados con la realidad, estimuladores y diversos. Por tanto, los docentes tienen que ser capaces de diseñar contextos y comunidades de aprendizaje, espacios físicos, temporales, socioculturales, relaciones humanas, interacciones, modos de hacer, en definitiva, actividades que permitan trabajar a cada aprendiz en el escenario de la escuela de manera personalizada y relevante. Los contextos hoy son virtuales y presenciales. No podemos concebir una escuela del siglo XXI exclusivamente con los espacios presenciales, necesitamos compartir, integrar y potenciar los espacios virtuales y los espacios presenciales. Los espacios virtuales no sustituyen en ningún sentido al docente, lo complementan y ayudan al desarrollo de las capacidades humanas fundamentales.

- Competencia para aprender a autorregularse y a desarrollarse profesionalmente a lo largo de toda lo vida. Tenemos que desarrollar en los docentes la capacidad de trabajar en grupo y aprender cómo aprender para asumir el destino de su desarrollo profesional futuro. El desarrollo profesional realmente relevante y que se corresponde con la personalidad requerida en la profesión docente, es aquel que parte de las propias necesidades y el propio convencimiento. Si el docente es capaz de autorregularse y aprender cómo aprender a lo largo de toda la vida, él mismo desarrollará la capacidad de desarrollo profesional docente necesario para afrontar los inevitables, sustantivos y vertiginosos cambios que la era digital impone en el conocimiento y en la vida contemporánea.

Para lograr estas competencias resulta fundamental que se consideren algunos lineamientos de carácter metodológico que, basados en la sistematización de los requerimientos del Reglamento de Régimen Académico del CES y los principios del Modelo Pedagógico de la UNAE, se constituyan en elementos de orientación de la práctica y configuran el deber o nuevo enfoque de la Carrera de EB:

- La concepción del proceso de formación profesional de la carrera de EB debe basarse en la integración sistémica de los procesos sustantivos universitarios: docencia, investigación, prácticas pre-profesionales y vinculación con la colectividad.

- La formación profesional de la carrera implica que todos y cada uno de los profesores, contribuyan a la plena comprensión de la realidad educativa propia de la EGB. Todo el accionar docente debe estar orientado a la transformación de este nivel educativo y asegurar su carácter inclusivo. Para ello deberemos incidir en la formación de una concepción de la EGB que, en correspondencia con sus particularidades, las que se deberán estudiar sistemáticamente por diferentes vías, tenga en cuenta su complejidad como fenómeno social; su carácter histórico concreto; su función social de transmisión y asimilación de la cultura (socialización) y de formación y desarrollo de la personalidad (individualización), así como el carácter interactivo, relacional, donde intervienen múltiples agentes e influencias.

- La práctica debe constituir el punto de partida y el fin del proceso de formación de los estudiantes. Esta debe desarrollarse desde los presupuestos de la investigación y la construcción de nuevos conocimientos, en el plano de la actividad académica, y de los procesos devenidos de las realidades profesionales y sociales. Su orientación y ejecución debe basarse en las direcciones siguientes: 
- Como forma de comprobación de los contenidos, de su validez.

- Para la aplicación de los contenidos teóricos, lo que facilita su apropiación desde las vivencias de situaciones profesionales reales y diversas.

- Como forma de adquisición de nuevos conocimientos y habilidades profesionales a partir de actividades investigativas que propicien el surgimiento de nuevas necesidades de aprendizaje y estimulen el desarrollo de la motivación y la identidad profesional.

- Como vía de sistematización de todos los contenidos, especialmente la formación de habilidades, valores y modos creadores de actuación propios de la profesión, que se correspondan con las necesidades de la EGB y del contexto profesional y social.

- Orientar todas las influencias de la carrera hacia la integralidad de la formación del profesional de la educación. La integralidad del proceso formativo de los estudiantes, con predominio del trabajo tutorial orientado al desarrollo de conocimientos, habilidades, actitudes, emociones y valores, así como el desarrollo de motivos, debe constituir la base para que los maestros en formación comprendan la cultura, se promueva su auto-realización y el desarrollo de su compromiso con la sociedad.

- Prestar atención a la cultura vivida y cotidiana como punto de partida para la construcción y reconstrucción de las identidades. Se debe propiciar proyectos pedagógicos que posibiliten el seguimiento a las trayectorias personales de los estudiantes basados en la experiencia, necesidades y potencialidades de cada uno, como base para proyectar procedimientos que potencien su desarrollo.

- Priorizar el proceso de formación de nuestros estudiantes desde una perspectiva interdisciplinar.

- Asegurar la contextualización del aprendizaje en todas las asignaturas y demás procesos en que participan los estudiantes, como vía de proyección de contrastación teórica en los entornos y ambientes de formación propios del contexto profesional de la EGB y social en que transcurre el proceso de formación.

- Propiciar la incorporación de acciones educativas que impliquen la colaboración en la producción, aprendizaje y socialización de los conocimientos y saberes, así como el desarrollo de habilidades para diagnosticar problemas, comprender, interpretar y elaborar propuestas de solución y la superación de las tensiones de la realidad, así como para retroalimentar y evaluar estos procesos.

- Procurar la organización de los aprendizajes a partir del empleo de métodos basados en la autorreflexión crítica, individual y colectiva, desde un enfoque complejo, a partir de considerar que el estudiante ya posee estructuras cognitivas, un capital cultural previo, y es protagonista y responsable de su propio aprendizaje.

- Asegurar que los estudiantes se enfrenten a reflexiones teóricas que provoquen confrontaciones, desde la que interpreten y reinterpreten la realidad que estudian. En este proceso de producción y adquisición de los conocimientos y saberes, los estudiantes han de tener libertad para expresar sus ideas y defender sus puntos de vista, establecer nuevas relaciones en el seno del grupo, quien sugiere nuevas formas de trabajo y evaluación. Debe asegurarse enseñar-aprender a pensar, analizar e inferir como contenido del proceso.

- Asegurar el empleo eficiente de las tecnologías, la educación a distancia y otros que aseguren escenarios virtuales, los que en estrecha relación con los de aprendizajes presenciales conformen nuevas formas de organización de los aprendizajes.

Estas características, como nuevo enfoque a lograr mediante el proyecto de investigación referido, determinan un nuevo horizonte epistemológico a asumir como criterio básico para el perfeccionamiento de la carrera de EB en la UNAE y la formación profesional de sus egresados. El mejoramiento e integración de los procesos universitarios en la formación de los futuros profesionales de EGB "revelan un cambio respecto a la práctica universitaria que generalmente se aplicaba en las universidades en el Ecuador y expresan los elementos fundamentales que guían la transformación para alcanzar el deber ser del proceso de formación". (13) 


\section{Conclusiones}

EL proyecto contribuirá a reforzar teóricamente criterios pedagógicos relevantes, en tanto propondrá una concepción pedagógica integradora diferente a la que actualmente rige la formación profesional de EGB en la carrera de la UNAE. Esta concepción incluye un sistema de acciones concebido científicamente en el que se contextualizará la sistematización teórica del proceso de formación profesional y el desempeño profesional pedagógico y su mejoramiento en la carrera. De igual forma, se determinarán las relaciones existentes entre las dimensiones e indicadores en que ha de expresarse la formación profesional pedagógica de los estudiantes de la carrera, y se precisarán las principales tendencias transcurridas y existentes en ese proceso en el Ecuador y su correspondencia con las actuales exigencias del Sistema de Educación Superior del país y los requerimientos del Modelo Pedagógico de la UNAE.

Desde el punto de vista práctico, el proyecto aportará una estrategia para la implementación de la concepción pedagógica en la carrera de EGB, validado teóricamente como respuesta a las problemáticas organizativas y curricular existentes; ello ha de favorecer los procesos sustantivos en los que participan los estudiantes y, en consecuencia, contribuirá a elevar la calidad de los procesos formativos de la Universidad.

La actualidad del proyecto está dada en que los elementos teóricos, metodológicos y prácticos de la concepción pedagógica y el sistema de acciones que incluye, deben posibilitar realizar los ajustes necesarios para el mejoramiento del proceso de formación profesional de la carrera, tal como se requiere de acuerdo a los requerimientos pedagógicos del Modelo Pedagógico de la UNAE y las exigencias del Sistema de Educación Superior del Ecuador.

\section{Referencias Bibliográficas}

1. Universidad Nacional de Educación. Modelo Pedagógico de la UNAE. $2015 .$.

2. Universidad Nacional de Educación. Información de oferta académica. [Online].; 2016. Available from: http://www.unae.edu.ec/\#!oferta-acad-mica/wsglm.

3. UNAE. Proyecto de carrera: Educación General Básica. Azogues. Ecuador:; 2014.

4. Ministerio de Educación y Ciencia. La formación del docente universitario. Madrid. España:; 1992.

5. Sánchez J. Sánchez J. El desarrollo profesional del docente universitario. Universidad Politécnica de Madrid. España. [Online].; 2016. Available from: http:/www.lachacana.ec.

6. Añorga J, Valcárcel N. La Profesionalización y Educación Avanzada Sucre. Bolivia; 1995.

7. Pérez AM. El entrenamiento metodológico conjunto para la profesionalización de los jefes de departamentos de las secundarias básicas. Tesis en opción al Grado Científico de Doctor en Ciencias Pedagógicas. La Habana. Cuba:; 2000.

8. Añorga J, Robau D, Magaz I, Caballero A. Glosario de Términos de la Educación Avanzada. La Habana. Cuba:; 1995. 
9. Moreno V. El desempeño profesional pedagógico de los profesores de educación secundaria de la ciudad de Veracruz, México. Resumen de tesis en opción al Grado Científico de Doctor en Ciencias Pedagógicas. La Habana. Cuba:; 2003.

10. Hernández X. Evaluación del desempeño profesional de los estudiantes de la Licenciatura en Educación Preescolar. Tesis en opción al Grado Científico de Doctor en Ciencias Pedagógicas. La Habana. Cuba:; 2003.

11. Larrea E. Modelo Educativo. Fundamentos Epistemológicos, Políticos, Antropológicos y Filosóficos. Guayaquil. Ecuador:; 2014.

12. Universidad de Texas. El Proyecto Tejas PRIMERO - Un proyecto de La Red de Familia a Familia. [Online].; 2012. Available from: www.texasprojectfirst.org/GlossaryCsp.html.

13. Portela R, Cisneros P. La integración de los procesos sustantivos en la formación profesional. Propuesta de una concepción pedagógica en sintonía con las transformaciones de la Educación Superior Ecuatoriana. In Memorias del X Congreso Internacional Universidad 2016; 2016; La Habana. p. 1732-1742. 